\title{
Reaching out for Quality: Considering Security Requirements in the Design of Information Systems
}

\author{
Hubert F. Hofmann and Ralph Holbein \\ Institute for Informatics, University of Zurich \\ Winterthurerstr. 190, CH-8057 Zurich, Switzerland \\ email: \{hofmann, holbein\}@ifi.unizh.ch
}

\begin{abstract}
Security requirements are a fundamental ingredient for an information system's quality. Despite their importance, security requirements play the role of a "stepchild" in software engineering. If considered at all they cover the technical dimension of information systems, i.e. the electronic part of information processing. This view is insufficient to deal with the requirements of the "real world", i.e. the organisational practice. It is not just the technical criteria which are decisive in specifying security requirements. We have extended these criteria to incorporate the social and the economic dimension of information exchange in organisations. We will illustrate this extension of traditional approaches in a comprehensive security framework and we will demonstrate the interaction of the additional security criteria with traditional approaches.
\end{abstract}

\section{Introduction}

Quality is a multi-faceted concept. Depending on the experiences and expectations of the people involved, the quality of information systems is defined by various characteristics. The characteristics making up the quality of an information system depend both on its technical implementation and on its working environment. In other words, when defining characteristics like security, efficiency, usability, and portability for an information system, we also have to consider its organisational context. Defining these characteristics, often called non-functional requirements, is part of the requirements analysis (RA) process; not an "add-on" when the system is already in productive use [15]

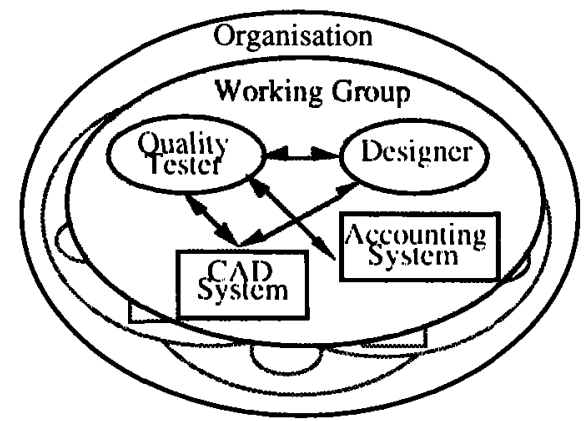

Fig. 1: Information system quality: schematic representation of the different aspects of information system quality. There are three levels: product, individual, and organisation. Details: See text. 
Quality Aspects. The various aspects of quality are depicted in Figure 1 . The most prominent quality measures concern the technical information system (e.g. CAD system, accounting system). The term verification denotes those measures that are available at this level. Verification is the proof of consistency between two formal descriptions, typically the consistency of the programs with its specification, or the internal consistency of the specification against the demands of the specification language. Various standard verification techniques exist, e.g. [33] While verification operates between two formal domains, validation operates between an informal domain and a formal one. Validation focuses on the questions such as: Does the information system fulfil its quality goals? To be valid a system's specification has to describe the desired system appropriately, i.e. the specification has to be "correct" concerning the requirements. If the validation process is inadequate, misunderstandings and errors are propagated throughout the development process, and expensive modifications of the information system are later required to correct them.

Less prominent quality measures concern areas where non-functional requirements must be validated. First, at the individual and group level, there are quality measures of system-user interaction. Quality measures taken at this level relate to the field of HCI (Human-Computer Interaction). Also work psychological aspects, e.g. motivational factors or user satisfaction with the system must be considered. Second, we have to consider the information system in the larger context of the whole organisation. Measures taken at this level to ensure quality are derived from organisational theory $[6,18]$. It should be noted that an assessment at the organisational level is normally not considered part of a particular project, but the result of strategic business and information management.

In this paper, we will focus on one particular quality characteristic: security requirements. They address threats considered as relevant and directed to valuable objects. Their goal is to define appropriate security measures, so that the resulting risks are deemed acceptable [17]. Despite their importance, security requirements play the role of a "stepchild" in software engineering. Traditionally, they exclusively cover the technical part of information processing. In the traditional view, loss of confidentiality, integrity and availability are the basic threats. However, they do not deal with those threats that result from the IS's productive use and its working environment. Hence, we will extend the "basic" threats to achieve a more comprehensive view of information processing. We encompass the system's environment and consider activities which take place outside the technical information system. In other words, we take the use, impact and influence of information on decisions and human actions in an application domain into account. We will identify security criteria for the information exchange in and between organisations. These security criteria constitute a framework that guides the specification of security requirements and the systematic derivation of security measures.

\section{Requirements Analysis - An Exploration Cycle}

Requirements Analysis (RA) starts when a problem has been perceived that offers some potential for an information system and when it has been decided to tackle the problem this way. In our case, the use of computer systems for the exchange of information in and between organisations. It is difficult to specify the correct and complete specification at the beginning of RA. The "right first time" principle will only work, if at all, for simple problems. Complete knowledge about a problem 


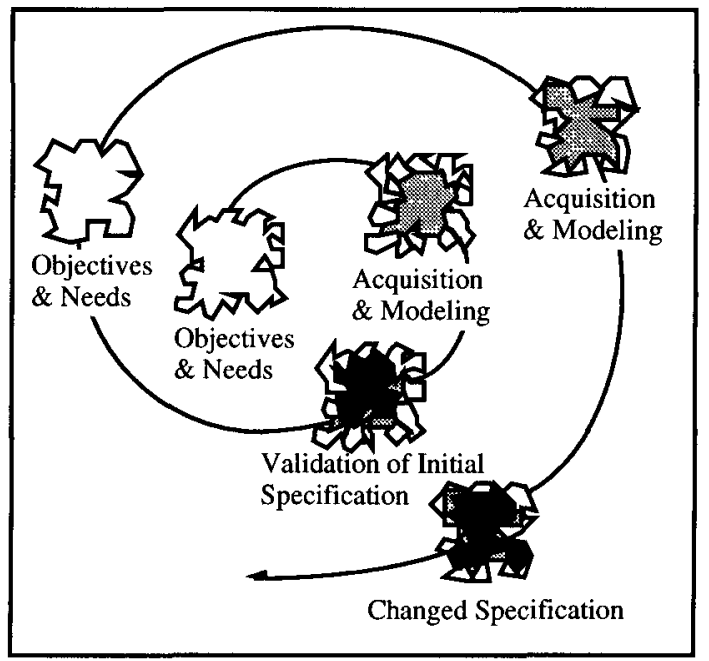

Fig. 2. Requirements Engineering Process

situation occurs in "artificial" situations only. In reality there are some doubts what the actual problem is, which solutions are available, and which solution should be selected (Fig. 2).

We understand RA as a cyclic process of exploring the perceived problem (objectives \& needs), proposing alternative solutions (acquisition \& modeling), and validating them (validation). This cyclic process comprises functional and quality (non-functional) requirements and allows the client to receive feedback on the analyst's interpretation of requirements and to correct misunderstandings as early as possible.

RA defines the boundaries of the proposed system by elaborating needs and objectives $[26 ; 7]$. The objectives for the information system are usually defined by (strategic) management, whereas the needs or the "wishes" of the client for the system are subject to debate. Quality requirements are usually formulated as goals and security requirements are an important component of quality goals, considering both organisational and individual needs. Elaborating objectives and needs and requirements acquisition and modeling are interrelated. The purpose of acquisition and modeling is to describe the requirements using informal, semiformal and formal notations. By acquisition and modeling we mean that individual(s) or group(s) identify or create requirements specifications together. Specifications are developed with the help of whatever sources are available, e.g. repositories, experts, textbooks, existing implementations. Candidate specifications must then be validated.

Analysing this process, several key issues can be identified [14]. In what follows, we will explain these issues and illustrate them from the viewpoint of security requirements.

Communication Barriers and Tacit Knowledge. Formal communication barriers among project teams and their members often hinder the understanding of requirements. Such barriers create a need for individuals to span team boundaries and to set up informal communication networks. While organisational changes can solve these problems, we are facing more fundamental communication barriers in RE: People know more than they can ever tell (learning by doing). When this "tacit knowledge" concerns a proposed information system, we are often at a loss as how to 
bring forth this knowledge. The problem is more than just a vagueness of the client concerning requirements, because tacit knowledge may not be describable by the client. It is an open question whether elements of our tacit knowledge are inaccessible in principle, or just hard to verbalise, and remain either consciously or unconsciously suppressed $[19,18]$. In the case of security requirements, we have to deal with a multifaceted, abstract concept. Security requirements cannot be discussed by "putting one's hands on the relevant threats." They exist in the minds of the people and therefore cannot be made explicit completely.

Multiple Views of Involved People. Information systems usually have a diverse client community and many software engineers are usually engaged in an information system project. Different people have different priorities, and view different requirements with varying degrees of importance. Also, customers and users are rarely the same people. Customers may impose requirements because of organisational and budgetary constraints, which are likely to conflict with user requirements. Taking different and potentially conflicting views into account reveals how the application domain is framed by the perceptions of people as opposed to being defined as objective facts. The comparison of these perceptions helps to investigate the advantages and limitations of each viewpoint. It shows, for instance, in what kind of situations users are performing and the way practice is distributed among them. Moreover, the use of multiple views prevents each party from monopolising the decisions on what to represent and what not to represent in the system [13, 9]. In specifying security requirements multiple views are absolutely necessary, because security is a concept, which is subjective in nature. Its subjectivity refers to the relevant threats, valuable goods and to the risks deemed acceptable.

Specification Integration. The emphasis shifts from designing and implementing a system from scratch to choosing and combining existing software components. Few information systems are built from scratch. In practice many projects extend an existing information system. Thus, the requirements analysts must understand these systems and the underlying requirements, which are often implemented by an integration of components from multiple vendors. Because existing components impose constraints, it is even more important to map between their formal specification and the requirements of the information system under consideration. Note that an immediate mapping from requirements to system implementation would lead to changes in the implementation, but not in the specification. This would cause the information system to diverge from the specification and the implementation would no longer reflect the requirements as stated by the client.

Specification Evolution. The introduction of information system will not only change the environment in which users work, but also their perceptions of it. As developers we have to be aware that this will happen and should observe and discuss the changes that information system has brought. Change does not only occur when an information system is introduced and put into practical use, but also when the environment changes (new insights into problems, new machines being installed, etc.). The need for change is exemplified by the countless adaptations an information system undergoes during its productive use. Although there are many reasons for the difficulty of maintaining information systems, lack of thorough attention to requirements analysis is a major problem, e.g. [23]. One reason for inadequate information systems is that the initial specification often is also the final one, thus hindering the communication and learning process of the stakeholders which might lead to more suitable information systems. Concerning security requirements, we protect the information system against threats which are explicitly considered in the 
specification. As the environment and the information system changes we have to adapt the specification to changing security requirements. To keep track of this process, we validate security requirements by "spot-tests" over extended periods of time.

In what follows, we will show the technical, individual and organisational dimensions that must be considered within a high-quality RA process, when dealing with security requirements for information exchange in and between organisations. Thus, we encompasses the exchange of information about the organisation itself, the business and know-how between members of organisation(s) or technological systems for the purpose of co-operation and division of labour, and the establishment of legal binding contracts or the co-ordination of semi-automated processes in the working environment. We describe these aspects as the components of a security framework (security criteria) which forms the basis for our ongoing research: the development of a comprehensive management of IT-security.

\section{Security of Information Exchange in Organisations: Relevant Dimensions}

In analysing the exchange of information in commercial areas, we have identified three basic dimensions: social interaction, economic potential, and technical data exchange. While the technical dimension and the social dimension are established in interpersonal communication [32], we will introduce the economic dimension as the third dimension of information exchange in organisations.

Characteristic properties of the social dimension (social interaction) are the creation of obligations and assertions between communication partners (Figure 3). Considering security requirements, relevant threats are: Loss of "Authenticity of the communication partners" and Loss of "Obligation to the content of the interaction." The economic dimension concerns the economic potential given by information and depends on its relevance to fulfil a particular task. In this case, security requirements

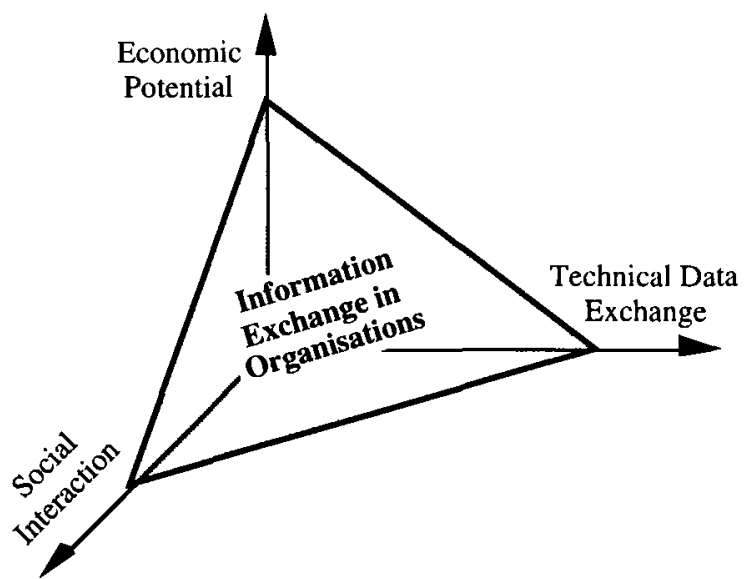

Fig. 3: Dimensions of information exchange. The social interaction comprises, for example, assertions and legally binding obligations. The economic dimension denotes the potential for the generation of economic benefits and the technical dimension focuses on the transmission of data. 
are only concerned that the information will be used for the intended goals of the organisation and that it builds a valid basis for the activities of the recipient. Relevant threats are: Loss of "Authenticity of the content" and "Misuse of information bound to intended goals." The technical dimension for secure information exchange in organisations covers the transmission of data between communication partners. Relevant threats are: Loss of "Confidentiality," Loss of "Integrity" and Loss of "Availability of the data and services" and also Loss of "Authenticity of the communication partners."

Traditional IT-security primarily covers the technical dimension described above. In the case of information exchange, security requirements for confidentiality are reduced to the question of data security, i.e. to prevent against unauthorised data access or disclosure and the misuse of organisational resources for data processing. These criteria allow the specification of an appropriate level of confidentiality concerning a third-party (Security for the OSI Reference Model: ISO 7498-2, ECMA, Bell/La Padula, etc.) $[7,8,19,20]$.

While the social dimension has been considered to some extent (e.g. nonreputation of communicative acts, legal aspects of electronic data interchange), the economic dimension, i.e. preventing the misuse of information in the case of conflicting goals, has not been considered up to now. Hence, this dimension must be considered also to ensure the confidentiality of communication partners. That is, one must differentiate between the issue of access control to data and the possibilities for the use and misuse of information which can be acquired. While the first one ensures authorised data access, the second one considers its intended use when granting authorisation. The important questions for identifying security requirements in this area are: What possible uses of the information exist? Which overall goals does the information support? What kind of misuse is possible?

Thus, we integrate three dimensions to specify security requirements. We consider the misuse and "incorrectness" of information, and the neglecting of obligations:

(1) Misuse of information: The sender has to deal with threats resulting from an inadequate behaviour of the recipient. We talk about inadequate behaviour when the information provided by the sender enables or causes non-intended actions of the recipient, i.e. actions that do not correspond to the sender's goals or interests.

(2) Incorrectness: The recipient has to deal with the threat that he will be guided into the wrong direction. That is, the provided information is not trustworthy (correct) and thus inhibits valid decision making.

(3) Neglecting obligations: For communication partners always exists the risk that one of the communication partners is unwilling to fulfil the obligations (e.g. a legal agreement) emerging out of the social interaction.

\section{The Framework}

The integration of the social, the technical and the economic dimension provide a basic structure for the identification and classification of security criteria [16]. These security criteria enable us to structure the multiple views of the involved parties, therefore reducing communication barriers and also supporting the integration of the multiple views.

The technical dimension of this framework considers the security criteria that result from the technical realisation of the information system. The technical 


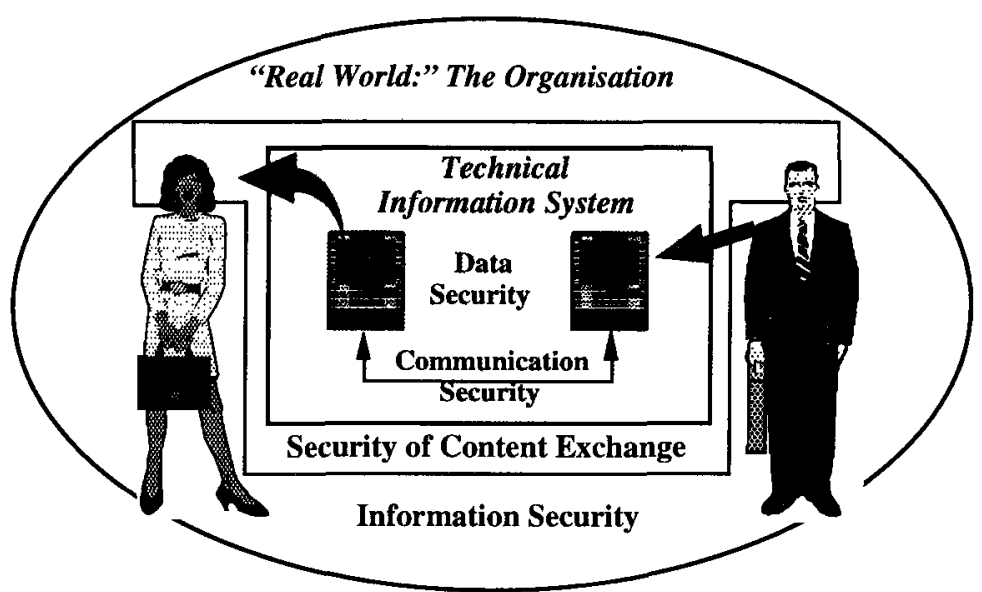

Fig. 4: Security levels of information processing

dimension alone is insufficient to cover the security requirements of information systems, because technical data exchange does not cover the essential phenomena of information processing. While information processing can be based on technical data exchange, it also takes place in the system's environment, i.e. in the "real world." Consequently, we also have to consider the social and the economic dimension of information processing to achieve the goal of a correct and complete specification of security requirements for information exchange.

Thus, our framework includes both of the following areas [17]: (1) Security of the technical communication processes realised by IT-systems (hardware, software and data). (2) Security of subsequent IT-external processing, i.e. the acquisition of content and information by individuals. Here we are concerned with (2a) relationships between the communication partners, e.g. emerging obligations, (2b) physical and cognitive activities which are enabled by the acquisition of information and by the extension of knowledge.

Figure 4 depicts the security levels of information exchange: (1) communication security including data security, (2a) the security of content exchange and (2b) information security form the generic levels for secure information exchange. Information systems are grounded in the workplace through the data and information they maintain. They represent the knowledge of one or more persons in the application domain. These persons are involved in a continuous interaction with a changing environment, make sense of this environment, and take action in it. When acting humans interpret and use the current situation as perceived. They are situated agents $[3,15]$, humans act in the "real world" and can communicate with each other using technical information systems. The technical information system provides data processing and transmission (data and communication security). The data exchanged by the technical information system and their sense-making, i.e. the content ascribed to the data, forms the cross point between the technical information system and the "real world." The interpretation process necessary for this last step normally takes place outside the scope of the technical system. Securing the content exchange ensures that the social interaction, which has been established between the communication partners by acquiring the data's content, is conform to a set of rules (e.g. law, group norms, organisational roles). Information security finally covers the 

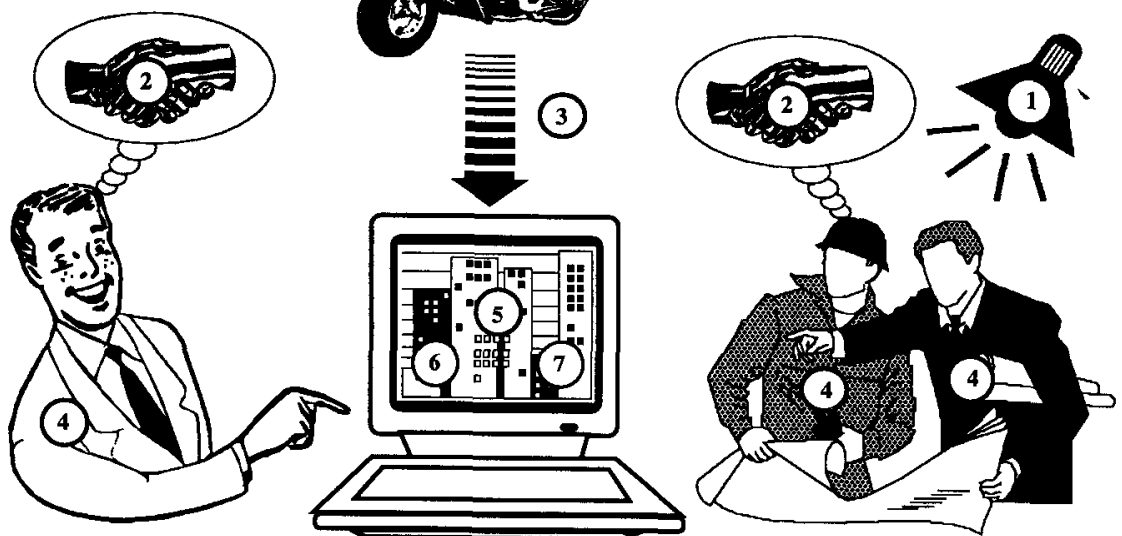

Fig. 5: Components of a comprehensive information exchange security framework: (1) goal conformity; (2) obligation; (3) authenticity of content; (4) authenticity of communication partners; (5) confidentiality; (6) integrity; and (7) availability are the security criteria that have to be considered when specifying the requirements for secure information systems. For more details, see text below.

economic dimension of information exchange, i.e. the goal-oriented behaviour made possible as a result of the communication.

Considering security requirements on all these levels of information exchange, we can define seven security criteria that are essential for specifying secure information systems [17]. All these security criteria describe basic threats regarding information exchange and hence their consideration is absolutely necessary when specifying secure information systems.

To explain the seven security criteria, we will use the following example: The design information for high-tech engines is one of the most valuable resources in the motor industry. We assume that designers develop and maintain engines with the help of CAD systems. Designers are responsible for design information, i.e. they are the "owners" of the resource. To ensure the quality of the design process, the information concerning intermediary design artifacts (e.g. plans, prototype engines) has to be exchanged between organisational units. We will select the exchange of design information between a drawing office and a quality assurance department in a motor company to illustrate the seven criteria that constitute our security framework. In doing so, we consider the technical, social and economic dimension introduced in the previous section (Figure 5).

(1) Goal Conformity. Goal conformity prevents the misuse of information, i.e. it ensures that the use of information is bound to the intended goals of the owner/sender. It considers the influence of information on the relationship between what humans think and their resulting goal-oriented actions (what they decide and/or do). To guarantee goal conformity, damage caused by the misuse of information must be prevented. Misuse of information damages the organisation when information enables an individual to act in a way that contradicts with the organisation's goals. Threats 
arising from the possible uses of information depend on the questions: What could anyone do with specific information? What could a specific recipient do given their individual competencies? That means the recipient's interpretation of information is very important, especially when he or she has specific competence. Goal conformity extends the traditional IT-security criteria to meet the requirements of the application domain. It considers the economic relevance of information arising from human interactions.

In terms of our example, goal conformity means that the quality assurance department does not misuse the received design information, e.g. they only use it to perform quality tests. So goal conformity ensures that the members of the quality department may only act according to organisational goals, when receiving design information. In other words, the boundaries of acceptable behaviours are given by the "spotlight" of organisational goals (Figure 5).

(2) Obligation. The exchange of information between communication partners includes social interaction. From the security point of view, we require that the obligations emerging from this interaction are binding to the communication partners, i.e. they accept the obligations. Their binding character is based on the kind of interaction, the legal systems, organisational policies, commitments or guidelines. For example, if a social interaction is documented as a legally binding contract, then obligations are provable and therefore enforceable in a social context $[11,12]$. Like goal conformity, binding obligations are independent of the realisation of technical information systems and are an extension of traditional IT-security criteria.

In our example, the primary reason for communication is to ensure the quality standard during motor manufacturing. Hence, communication establishes the following obligations: The designers have to provide the "right" information, i.e. necessary information which enables performance of quality testing, and members of the quality department have to perform the quality test adequately as negotiated.

$(3+4)$ Authenticity. Authenticity of communication partners is a widely accepted security criterion for information systems. It stems from the nature of interpersonal communication. Authenticity requirements encompass the authenticity of communication partners (as is the case of KERBEROS [26]) as well as the authenticity of a message's content, i.e. correspondence between representation and reality. Often the latter is not considered ${ }^{1}$ or discussed in the context of integrity. Authenticity of the content means that the representations can be validated according to the organisational "reality". In this sense, authenticity guarantees that valid decisions can be made based on the content of the system model.

In our example, the authenticity of content ensures that the design information adequately maps "reality," i.e. the design information precisely describes the "real" engine. The authenticity of communication partners ensures that the designer's communication partner is really the one who is intended to receive the design information and in turn this communication partner can be sure that the received information has been sent by the designer.

(5) Confidentiality. Confidentiality of data is a traditional IT-security criterion. It describes the state of being private or secret, i.e. only accessible by authorised people $[25,5,1]$. Unauthorised disclosure of data is a violation of confidentiality. Confidentiality is ensured by data access controls or encryptions in technical systems. Access controls are classified as either mandatory access controls that specify user

1 An exception is the Clark/Wilson model [4]. 
privileges and object classifications (e.g. top-secret, private, public) or discretionary access controls that define discrete relationships between an active and a passive component $[5,1]$. The more flexible techniques to access control are based on userrole descriptions $[21,30,22]$. To formally specify the underlying authorisations, various approaches are proposed in the literature [31,27, 28]. Steinke, for example, uses a group security model which describes access rights through a teamworkoriented organisational model [27]. Another way to ensure confidentiality is data encryption which uses cryptographic methods to preserve against the unauthorised interpretation of data. There are two major methods for data encryption: (a) symmetric encryption, i.e. encryption and decryption using the same key; and (b) asymmetric encryption, i.e. public and private keys for encryption and decryption respectively.

Considering confidentiality in our example, the technical information system has to guarantee that the data representing the design information is not accessible by unauthorised persons.

(6) Integrity. Integrity, like confidentiality, is a traditional IT-security criteria [2]. The distinction between the integrity of the data and the correspondence between representation and reality is rarely considered when specifying security requirements. We call the correspondence between representation and reality the authenticity of content (3). Integrity, as we define it, relates to the internal state of data and depends on the technical realisation of the system in use, but not on the system's representation.

Integrity, for example, prevents unauthorised and accidental modification of the data representing design information as stored or while transmitted.

(7) Availability. Availability is a state of existence or readiness for the resources and services (infrastructure, data, services) belonging to a technical information system. Availability is a basic requirement that is barely considered in existing security models [25].

In our example this security criterion guarantees that the data representing the design information is available when needed by authorised persons.

The criteria (1) goal conformity, (2) obligation, and (3) authenticity of content correspond to informal phenomena. Thus, these criteria specify quality requirements that must be validated in the first place. The security criteria (4) authenticity of communication partners, (5) confidentiality, (6) integrity, and (7) availability can be formally specified and thus are under the reign of verification.

\section{Managing Secure Information Exchange}

Corresponding to the suggested framework, we briefly describe those requirements an information system in productive use has to fulfil to achieve secure information exchange.

- Classification of the economic potential of information to be exchanged within a communication context, i.e. which activities of the communication partners are intended and which are possible? etc. Additionally, a classification of the communication partners is necessary: What organisational roles do they have? Which roles are possible based on their individual competence? etc.

- Classification of the type of interpersonal interaction. The type of interpersonal interaction refers to the obligations which arise out of this interaction. 
- Classification of the overall sensitivity of the data for loss of confidentiality, integrity, and availability.

- Anticipation of threats based on the previous classifications. Example threats are misuse of information, neglecting obligations, and disclosure of data. The probability of misuse can be anticipated, for instance, by using a model of goal-based human behaviour [24]. Although the risk of opportunistic behaviour is quite common in organisations, behaviour is not completely arbitrary, but governed by norms and rules. Acting depends on the situation, the individual's competence and on the available information to be exchanged. Usually the goals of the communication partners overlap but are not congruent. Their differences must be anticipated to discover the conflicts of interests arising from the exchange of the information.

- Selection of measures appropriate for preventing anticipated threats. That is, the selection of security measures to be used for data access and transmission, e.g. document exchange; and the selection of security measures to prevent the misuse of information, to establish obligations and to guarantee the correctness of the content.

\section{Conclusion}

A comprehensive security framework must consider the interdisciplinary aspects of socio-technical systems (e.g. the technical information system within an organisation). Thus, our security framework for information exchange within and between organisations considers the social, the technical and the economic dimension of an information system, i.e. (a) the security requirements specific to the basic threats concerning valuable objects of the application domain, and (b) the security requirements arising from the use of technical systems which have to be dealt with in general. The primary focus of this paper has been to illustrate the implications of this point of view when specifying security requirements.

We discussed the subjectivity of security (valuable objects, relevant threats and acceptable risks) and proceeded to explain the security relevant dimensions of information exchange. Then we described the components (security criteria) of a framework for information exchange. This security framework constitutes the basis for a systematic analysis of security requirements. According to its structure the framework guides the formal specification of requirements. That is, the framework shows the contribution of existing approaches to the overall security of information exchange $[31,27,28]$. Up to now existing approaches for secure information systems do not consider the economic dimension appropriately.

Moreover, economic, social and technical security criteria form a basis for the flexible application of security measures to achieve overall security. For that, contextual properties have to be defined to allow the anticipation of the mutual threats concerning communication partners (economic potential of information, intended interpersonal relationships, etc.). Then we can select a set of security measures which are completed by measures for preventing unauthorised data access and modification. Finally, the security levels of our framework and their structured set of security criteria support the quantitative specification of the risks underlying information processing in the application domain. 
Further work will be done in this area by the OBS project [29] and the Requirements Engineering project [10]. The goal of the OBS project is to realise security management that is flexible and enables the economic use of security measures. That is, we are developing a method for bridging the gap between the security requirements for information exchange and their formal representation.

Acknowledgements. We would like to thank Johannes Geiger, Andrew Hutchison, and Volker Stadler for discussing this article and suggesting improvements. Further thanks for the support of this work in general go to Norbert E. Fuchs and Steffi Teufel. We also gratefully acknowledge the support of the Swiss National Science Foundation. The work of Hubert F. Hofmann is partially supported by grant SNF 2132746.91. Ralph Holbein's work is supported by grant NFP/SPP 5003-34271.

\section{References}

[1] D.E. Bell, L.J. La Padula, Secure Computer Systems: Unified Exposition and Multics Interpretation. EDS-TR-75-30, The MITRE Corp., Bedford, March 1976.

[2] K.J. Biba, Integrity Considerations for Secure Computer Systems. TR-3153, The MITRE Corp., Bedford, 1977.

[3] W.J. Clancey, Situated Action: A Neuropsychological Interpretation. Cognitive Science, vol. 17, no. 1, 1993.

[4] D. Clark, D. Wilson, A Comparision of Commercial and Military Security Policies. Proceedings of the IEEE Symposium on Security and Privacy, 1987.

[5] Department of Defense, TCSEC - Trusted Computer System Evaluation Criteria. DoD 5200.28-STD, Department of Defense, USA, December 1985.

[6] W.E. Deming, Out of the Crisis. Cambridge, MIT Center for Advanced Engineering, 1986.

[7] ECMA, Security in Open Systems - A Security Framework. 46, European Computer Manufacturers Association (ECMA), 1988.

[8] ECMA, Standard ECMA-138 - Security in Open Systems - Data Elements and Service Definitions. Standard ECMA-138, European Computer Manufacturers Association (ECMA), 1989.

[9] A. Finkelstein, J. Kramer, B. Nuseibeh et al., Viewpoints: A Framework for Integration Multiple Perspectives in System Development. International Journal of Software Engineering and Knowledge Engineering, vol. 1, no. 2, 1992, pp. 31-58.

[10] N.E. Fuchs, Software Development Based on Executable Specifications. SNF 21-32746.91, Institute for Informatics, University of Zurich, 1993.

[11] R. Grimm, A Model of Security in Open Telecooperation. In: G.Neufeld, B. Plattner (eds.). Proceedings of the IFIP TC6/WG 6.5 International Conference on Upper Layer Protocols, Architectures and Applications, Vancouver, Canada, 27-29 May, 1992. 
[12] R. Grimm, A. Steinacker, Das Kooperations- und das Gleichgewichtsmodell Theorie und Praxis. VIS 93: Verlässliche Informationssysteme - GI-Fachtagung, München, Springer, 1993.

[13] R. Hirschheim, H.K. Klein, M. Newman, Information Systems Development as Social Action: Theoretical Perspective and Practice. OMEGA International Journal of Management Science, vol. 19, no. 6, 1991, pp. 587-608.

[14] H.F. Hofmann, Requirements Engineering: A Survey of Methods and Tools. TR 93.05, Institute for Informatics, University of Zurich, 1993.

[15] H.F. Hofmann, R. Pfeifer, E. Vinkhuyzen, Situated Software Design. In: Proceedings of the Fifth International Conference on Software Engineering and Knowledge Engineering San Francisco, Knowledge Systems Institute, 1993, pp. 622-628.

[16] R. Holbein, Informationssicherheit - Ein Blick über den Tellerrand der (Informations-) Technologie. European Conference on Computer Science, Communication and Society: A Technical and Cultural Challenge, Neuchatel, Schweiz, 1993, pp. 161-172.

[17] R. Holbein, Secure Information Exchange in Organisations. OBS-Report, University of Zurich, 1994.

[18] M. Imai, Kaizen: The Key to Japan's Competitive Success. New York, Random House, 1986.

[19] ISO, ISO: Security Frameworks Overview. International Organisation for Standardization, 1991.

[20] ISO, WD 10746-1: Reference Model for Open Distributed Processing ODP Part 1: Overview. WD 10746-1, International Organisation for Standardization ISO, 1992.

[21] J.I. Jones, M. Sergot, Formal Specification of Security Requirements using the Theory of Normative Positions. Computer Security - ESORICS 92, Toulouse, France, 1992, pp. 103-121.

[22] D. Jonscher, K.R. Dittrich, A Formal Security Model Based on an ObjectOriented Data Model. TR 93.41, University of Zurich, Institute for Informatics, 1993.

[23] F. Lehner, H.F. Hofmann, R. Setzer et al., Maintenance of Knowledge Bases. Fourth International Conference on Database and Expert Systems Applications, Prag, Springer, 1993, pp. 436-447.

[24] A. Newell, The Knowledge Level. AI Magazine, vol. 2, no. 2, 1981, pp. 120.

[25] D.B. Parker, Neuformulierung der Grundlagen der Informationssicherheit. Datenschutz und Datensicherung, no. 11, 1991.

[26] J.G. Steiner, C. Newman, J.I. Schiller, Kerberos, An Authentication Service for Open Network Systems. Winter USENIX Conference, Dallas, 1988.

[27] G. Steinke, M. Jarke, Support for Security Modeling in Information Systems Design. Database Security, VI: Status and Prospects (A-21), Elsevier Science Publisher, 1993, pp. 125-141. 
[28] R. Strens, J. Dobson, How Responsiblity Modeling Leads To Security Requirements. Department of Computer Science, University of Newcastle, 1993.

[29] S. Teufel, Offene Bürokommunikation - Sicherheitsmanagement im innerund zwischenbetrieblichen Informationsaustausch (OBS). NFP/SPP 500334271, Institute for Informatics, University of Zurich, 1993.

[30] T.C. Ting, S. Demurjian, M.-Y. Hu, Requirements, Capabilities, and Functionalities of User-Role Based Security for an Object-Oriented Design Model. Database Security, vol. 5, 1992.

[31] T.C. Ting, S.A. Demurjian, M.-Y. Hu, A Specification Methodology for User-Role Based Security in an Object-Oriented Design Model. Sixth Working Conference on Database Security, Burnaby, IFIP WG 11.3, 1992, pp. 351378.

[32] T. Winograd, F. Flores, Understanding Computers and Cognition. New York, Ablex, 1986.

[33] John A. Wise, V. David Hopkin, Paul Stager (eds.), Verification and Validation of Complex Systems: Human Factors Issues. Berlin, Springer, 1993. 